\title{
EDITORIAL
}

nature

cell biology

\section{Policy matters, policies that matter}

Autumn seems a good time to take stock of some topical parts of the editorial process.Nature journal editors have always encouraged discussion of data before submission. Indeed, we regularly encourage submission to the journal after informal discussion, and we receive some of our best papers this way. All Nature journals accept presubmission inquiries through the online submissions system (http://mts-ncb. nature.com/). We provide feedback on formally submitted 'pre-subs' within a couple of days. Rapid response and ease of preparation probably account for the increasing popularity of this format currently over $20 \%$ of all submissions. However, a negative decision often leads to an attempt to further discuss and explain. Although we fully understand the validity of further discussion, the volume of submissions necessitates that we do not entertain further exchanges. If you feel certain that we have underappreciated your findings, we are happy to read a full submission that allows discussion of all aspects of the work and this undoubtedly makes for a more informed decision.

We are often asked if cover letters are essential. Indeed, it is sometimes evident from a cover letter that an author is expecting us to read no further. This reveals a significant under-appreciation of the thoroughness of the editorial process: Nature journal editors are expert scientists who assess all manuscripts thoroughly. Decisions are made only after significant editorial discussion and, if in doubt, we will consult informally with experts in the field. We save our authors' and referees' time by only reviewing manuscripts that, in our opinion, stand a realistic chance of publication. Cover letters are certainly helpful in framing the author's perception of their dataset, as well as to alert us to related data or individuals they wish to exclude from the reviewing process (we honour exclusions as long as they can be counted on one hand).

It is important to submit manuscripts that are close to our format guidelines, although it is certainly not necessary to fine-tune format requirements on initial submission. The decision whether to publish a dataset in our Article, Letter or Brief Communication format is given very careful editorial consideration, and this will usually be decided firmly only when a manuscripts is deemed publishable in principle.

Given the recent publicity surrounding the editorial retraction of a paper from the journal Cell (see 6 October editorial and The Scientist, September 29th 2005), we wish to restate our policy on refutations. We will consider substantive contributions that claim to refute a major finding published in this journal. We will publish a refutation only if its conclusions are of outstanding interest to our readers and only if the data passes peer review. We usually invite the author of the original paper to comment and we will co-referee this comment (see Nature Cell Biol. 7,433 (2005)). Substantial errors introduced by the authors or editors are redressed by posting corrigenda or errata, respectively. Addenda may be added at the editors discretion when additional information from the authors alters the interpretation of a paper. If a manuscript is fundamentally flawed or fraudulent, all or some of the authors, or the editors, may decide to retract the paper after due consultation.

We have previously discussed why we value our current system of confidential peer review (Nature Cell Biol. 5, 583 (2003)). Most other journals retain similar systems, although the role of academic editorial boards and the fraction of manuscripts refereed varies. Nevertheless, the debate about systems with more transparency and greater accountability continues.

\section{Reviewing peer review}

The latest experiment in biological publication is a new pan-biology journal named Biology Direct from BioMed Central (www.biologydirect.com). The author selects referees from the editorial board who then decide whether to review the manuscript in detail. This decision appears to amount to a decision to publish. The referees can also choose to publish signed comments alongside the manuscript. The key to an authoritative editorial process lies in constructive peer review. Biology Direct editors have rightly noted that forced publication of full signed reports from referees is ill-advised: top journals invariably require substantive revision before publication, thus, final referee reports only rarely contain a detailed scientific critique and earlier reports are essentially out of context as the accepted manuscript was revised to address them. Biology Direct states that published reports aim to "provide pointers as to the content and value of a publication". We agree that this is useful, and we have a long-established 'News and Views' section where experts, occasionally referees of the paper, present such pointers. Although there is only one News and Views mansuscript per paper, its analysis is thorough and developmental editing maximizes accessibility. Thus far the review process of Biology Direct does not diverge greatly, with the exception that referees are known (at least to the authors) and that they are drafted from the limited pool of the editorial board. We argue that this limits both the amount of technical expertise and editorial opinion to a finite group of people. We select the most authoritative experts while carefully avoiding potential conflicts of interest - we believe our system better avoids conflicts as we honour referee exclusion requests and explicitly ask for declarations of conflicts before review. Furthermore, impartial editors screen for possible biases in reports. We also argue that our editorial opinion is more informed by integrating the views of a handful of referees and editors. We have often considered signed reports, and indeed we allow this if the referee so desires. However, in our experience this tends to select against incisive critique: too much is at stake.

Finally, a call for fairness: we absolutely rely on constructive peerreviewing. Editors and referees should judge the dataset in hand, and not the reputation or record of the authors. We have come across cases where authors are 'ostracized' from a community and peers refuse to judge their work - the inevitable outcome is that decisions are less informed.

Links to previous editorials on policy matters can be found on Connotea (keyword: policies). 